\title{
A COMPREHENSIVE REVIEW ON PHARMACEUTICAL AND NUTRITIONAL APPLICATIONS OF INULIN
}

\section{SAI KISHAN ${ }^{1}$, AKHILA A. R. ${ }^{1}$, SUBHASHREE SAHO0 ${ }^{1}$, P. K. KULKARNI ${ }^{*}$}

\author{
${ }^{1 *}$ Department of Pharmaceutics, JSS College of Pharmacy, JSS Academy of Higher Education and Research, S. S. Nagara, Mysuru 570015, \\ Karnataka, India \\ Email: pkkulkarni@jssuni.edu.in
}

Received: 23 Dec 2020, Revised and Accepted: 13 Mar 2021

\begin{abstract}
Inulin is a versatile, water-soluble polysaccharide that is commonly available in nature. In the pharmaceutical industry, the non-digestible function of inulin has made it attractive. Inulin is granted with GRAS status by the FDA and more than 30,000 plants in nature store inulin as a carbohydrate. The chicory is the key plant source of inulin out of all sources. It can be used as the sugar or fat replacer in the processed foods to influence the desirable characteristics. Good biocompatibility, essential chemical properties, and a wide variety of bioactivities have rendered inulin an outstanding natural nutrient. Regulating blood sugar, antioxidant, anticancer is some of the biological activities of inulin. Inulin can also be a carrier for colon/tumor targeting, as only specific enzymes in the colon zhydrolyze the inulin. It allows the growth of micro-flora, the good bacteria in the gut. Inulin is considered as a prebiotic as it is fermented by bacteria that normalize the colon. This review offers an in-depth insight into its novel Pharmaceutical applications as well as sources, processing, physicochemical properties, and nutritional and physiological activities. The chemically modified inulin is gaining a specific interest in the pharmaceutical field with its outstanding properties which are discussed in this review.
\end{abstract}

Keywords: Inulin, Pharmaceutical applications, Targeted drug delivery, Prebiotics

(c) 2021 The Authors. Published by Innovare Academic Sciences Pvt Ltd. This is an open access article under the CC BY license (https://creativecommons.org/licenses/by/4.0/)

DOI: https://dx.doi.org/10.22159/ijap.2021v13i3.40863. Journal homepage: https://innovareacademics.in/journals/index.php/ijap

\section{INTRODUCTION}

More than 30,000 plants in nature store inulin as a carbohydrate. It was first isolated by Valentine Rosein 1804 from the roots of compositae plant-Inula helenium, and named inulin by Thomson in 1817. Inulin being a polysaccharide, belong to the carbohydrate class known as fructans [1]. Fructans are the structures in which the fructose (sugar) molecules are held together in the form of a chain with the aid of $\beta$ - $(2 \rightarrow 1)$-D-fructose-fructose bond. The current trend of Inulin is towards the food and pharmaceutical industries. It is found in a wide range of herbs, fruits, and vegetables, including onions, bananas, garlic, wheat, asparagus, and artichokes. Certain bacteria and fungi are also capable of producing inulin. Chicory or Jerusalem artichoke tubers are the current sources of raw materials for the industrial use of inulin because of their high inulin content. It can be used as the sugar or fat replacer in the processed foods to influence the desirable characteristics. The sweetness level of inulin is about 10 percent in comparison to sucrose. The good biocompatibility, significant chemical properties, and wide range of bioactivities made inulin an excellent natural nutrient.

Inulin does not only enhance the food flavor but also has different physiological functions, like reducing blood sugar and fat, including cholesterol and triglycerides. It is also used for constipation, diarrhea, diabetes, and as an anticancer [2]. Recent advances of inulin in the pharmaceutical field include colon-targeted drug delivery, as the inulin can only be hydrolyzed by the specific enzymes in the colon. It allows the growth of micro-flora, the good bacteria in the gut. Inulin is considered as a prebiotic as it is fermented by bacteria that normalize the colon.

Besides, inulin is inexpensive and readily available. In European Countries, inulin is recognized as a natural food ingredient [3]. Adding inulin to the diet will enhance the health of the individual as it is considered a functional food. Food, Nutrition, and Health Trends 2020 highlight the main market trends driving industry growth. 'As a natural sugar replacer, inulin became a success, used in several products, and its existence means that businesses can also mark the improved fibre quality on the label.

\section{Chemistry and structure}

Inulin is a polydisperse $\beta-(2-1)$ fructan. It is made up of residues of $\beta$-D-fructose bound together by glycosidic bonds [4]. At the end of each fructose chain, a glucose molecule is linked by $\alpha$-Dglucopyranosyl or $\alpha-(1 \rightarrow 2)$ bond, similar to sucrose [5]. Inulin is indigestible in the human small intestine due to $\beta$-configuration of anomeric carbon, however, can be fermented by the intestinal microflora in the large intestine. Its $\beta-(2-1)$ bonds are a special feature of the structure of inulin. These linkages inhibit the digestion of inulin like a standard carbohydrate and are responsible for its decreased caloric value and benefits of dietary fiber. The number of fructose units in chicory inulin ranges from 2 to 60 , representing a mixture of oligomers and polymers.

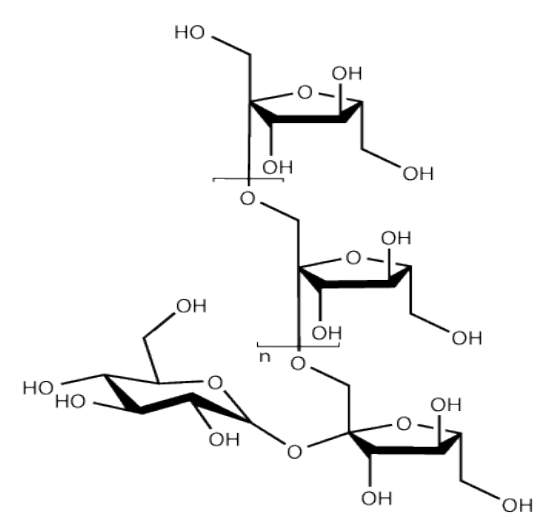

Fig. 1: Chemical structure of inulin [6]

Inulin is expressed as GFn, where G denotes glucose unit, F denotes fructosyl residue, and $\mathrm{n}$ is the number of fructose residues. Functionality and industrial applications of the inulin are affected by the degree of polymerization [7]. In functional foods, inulin can be used as dietary fibre and prebiotics with varying degrees of polymerization. The degree of polymerization in bacteria $(10,000$ to $100,000)$ is high compared to plants inulin $(<200)$, which is affected by the physical as well as climatic conditions of the plant.

The inulin is also called oligofructose when the degree of polymerization is low $(\mathrm{n}<10)$ [8]. Oligofructose resulting from 
chicory contains fructose chains (Fm) and fructose chains with terminal glucose units (GFn) whereas, Synthesized oligofructose contains only GFnmolecules. However, both express the same nutritional benefits. Short-chain inulin is primarily used to be a sweetener in food, while long-chain inulin is primarily used to be a fat replacer and improves texture.

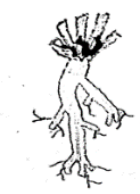

Chicory (Cichorium intybus) Dry root (64-78\%)

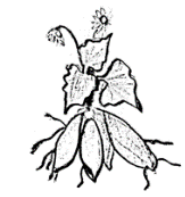

Yacon (Smallanthus sonchifolius) Wet root $(7-8 \%)$

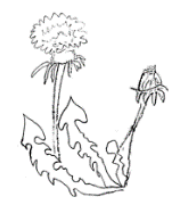

Dandelion (Taraxacum officinale) Root $(12-15 \%)$

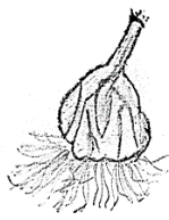

Garlic

(Allium sativum) Wet tuber $(12-24 \%)$ Dry tuber $(>70 \%)$

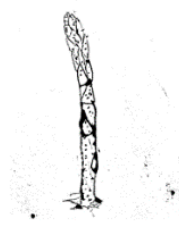

Asparagus

(Asparagus officinalis) Wet root $(2-3 \%)$

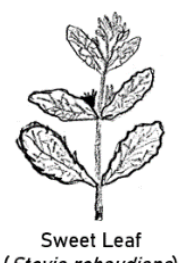

(Stevia rebaudiana) Leaf $(18-20 \%)$

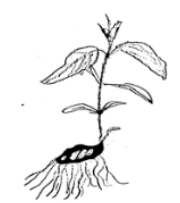

Jerusalem Artichoke (Helianthus tuberosus) Wet tuber $(8.6-13 \%)$

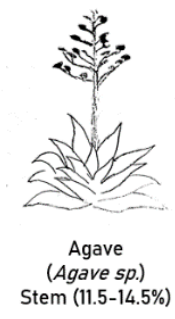

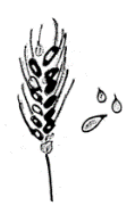

Barley

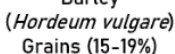

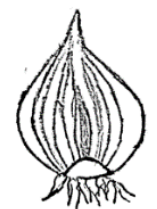

Onion

(Allium cepa)
Bulb $(2-8 \%)$

Fig. 2: Inulin content in various plant sources

\section{Production}

Inulin and Oligofructose, which is widely available on the market for industrial food products, is either synthesized from sucrose or derived from chicory roots. Large variety of plants naturally contains inulin [9]. Jerusalem artichoke and dahlia are reported as strong sources of industrial production in temperate regions [10]. Among all the parts, the root parts of various plant sources are rich in inulin and used for extraction. Inulin manufacturing resembles the process of sugar extraction. Initially, the roots are harvested, sliced, and washed. Followed by a hot water diffusion process, then purified and dried. A high-performance (HP) type of inulin is noted as a recent advancement in the production of inulin. By eliminating the shorterchain molecules, this substance is manufactured. This results in the elimination of both the residual sugars and oligomers. With no sweetness contribution, HP inulin has almost twice the fat mimetic properties of normal inulin.
Industrially most of the inulin is derived from chicory. It is a biannual plant belonging to the Asteraceae family. Extraction and initial purification of raw sugar are carried out in the first step, which is further processed during the second phase of production to obtain a commercial product (above 99.5 percent). In order to eliminate impurities in the purification process, the extracted juice involves several steps such as pre-liming, followed by liming and carbonation at a high temperature $\left(80-90^{\circ} \mathrm{C}\right)$, which may contribute to hydrolysis in the juice and may also inject additional calcium ions, requiring further treatment for purification like microfiltration [11]. In the inulin extraction process, some advanced technologies such as supercritical carbon dioxide, ultrasound, and pulsed electric field (PEF) are involved in achieving higher yields of the purified final product with lower energy consumption. The subsequent inulin contains a degree of polymerization ranging from 3 to 60 , mimics the original chicory.

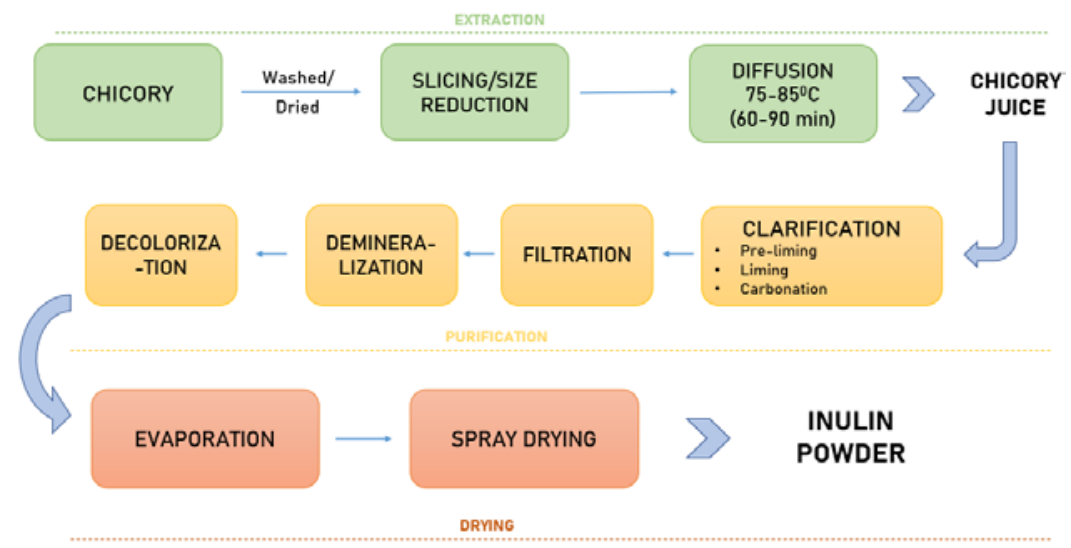

Fig. 3: Extraction of inulin powder from chicory [12]

\section{Physicochemical properties}

Chicory-derived inulin is a white powder that has increased clarity for fine particles and is odorless. The long-chain inulin has no sweetness, while standard chicory inulin has a sweetness level of approximately
10 percent relative to sucrose [13]. As a distinctive food ingredient, inulin provides many major dietary advantages along with some industrial properties. Inulin can decrease the freezing point of water and also can raise the boiling point of water. The average molecular weight of inulin varies in the range of $5600-6300 \mathrm{~g} \mathrm{~mol}^{-1}$. 


\section{Sweetness}

The chain length of the inulin decides the sweetness level. Shortchain inulin has a sweet taste, while long-chain inulin remains tasteless. It gives a pleasant feel without leaving any bad flavor in the mouth with its mild and non-irritating taste. Researches proved that Long-term inulin intake would not cause harm to the teeth. Further, inhibits microbial growth [14]. Inulin can act as a substitute for artificial sweeteners like aspartame.

\section{Solubility}

Inulin is partially dissolved in water almost 10 percent at $25{ }^{\circ} \mathrm{C}$ allowing it to be applied without any precipitation in an aqueous medium. To produce an inulin solution, the use of hot water at 50$100{ }^{\circ} \mathrm{C}$ is suggested [15]. Inulin being hydrophilic, highly soluble in hot water, and sparingly soluble in ethanol or cold water. The solubility of the inulin can be affected by crystallinity. At $23^{\circ} \mathrm{C}$, beta inulin is highly water-soluble. Other polymorphs need higher dissolving temperatures. In strongly acidic conditions, the $\beta-(2-1)$ bonds present between the fructose units can be partially hydrolyzed.

\section{Hygroscopicity}

The high hygroscopicity of inulin is an excellent feature in the food industry. Inulin has the capacity of adsorbing water, thereby reducing water activity. This feature can effectually slow the evaporation of water in food manufacturing to avoid product odor and extend the shelf-life.

\section{Viscosity}

The viscosity of the inulin solution is low and it depends on its concentration. At $10{ }^{\circ} \mathrm{C}, 5 \%$ solution shows 1.65 millipascal-second and $30 \%$ solution shows 100 millipascal-second. As the IN concentration exceeds $10 \%$, the viscosity starts increasing.

\section{Gel formation}

The inulin gel formulation is determined by many factors like polymerization, $\mathrm{pH}$, particle size, and the process of manufacturing. Wheninulin's concentration reaches $10-30$ percent; the gel starts to form [16]. The gel is formed immediately and very smooth when its concentration reaches 40 percent to 50 percent, while the gel created is quite firm above 50 percent. The gel formation rate increases with the concentration. For the formation of gel, a low degree of inulin hydrolysis and a critical concentration of inulin is necessary. Several inulin hydrogels are already designed for use as possible carriers for the release of variable composition drugs into the colon [17].

\section{Stability}

Inulin is not readily degraded even when it is heated at $100^{\circ} \mathrm{C}$. Hence it exhibits very good thermal stability. Owing to the lack of free water, IN gels are very stable and not readily hydrolyzed. Sigma crystalline inulin possesses higher thermal stability when compared to the other three forms of crystalline inulin. The thermodynamic stability of crystalline inulin falls in the order of beta<alpha<gama<sigma. Inulin is not suggestable for use in acidic beverages as it gets hydrolyzed at $\mathrm{pH}<4$

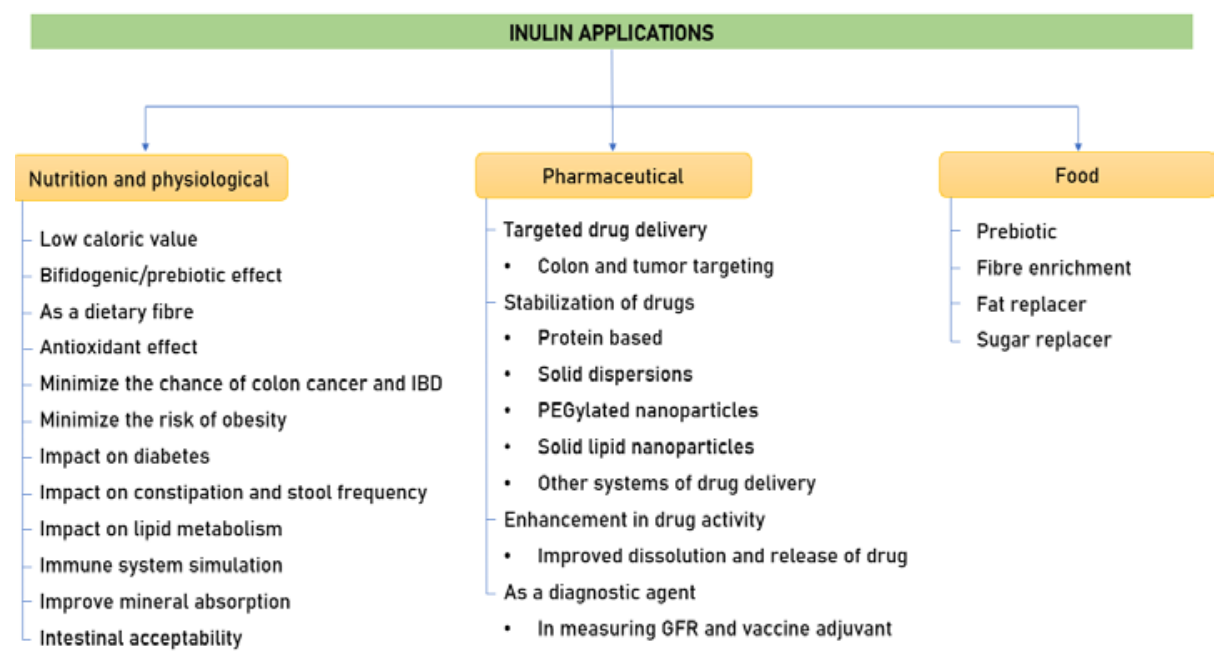

Fig. 4: Applications of inulin

\section{Nutritional and physiological activities}

\section{Low caloric value}

The nutritional properties of inulin and oligofructose are fascinating and satisfying. The low caloric value of inulin made it a choice to replace sugar or fat thereby reducing the calories in ice creams, baked products, dairy products, and certain foods. As human intestinal enzymes cannot digest the inulin, it passes the upper GIT without being metabolized and it is because of the $\beta-(2-1)$ bonds linking fructose molecules. The energy metabolism of the host organism can still be improved by short-chain fatty acids and lactate of inulin. These are produced as inulin is converted through the action of intestinal bacteria into short-chain fatty acids [18]. The caloric value of inulin is around $1-1.5 \mathrm{kcal} / \mathrm{gram}$. The other fermentation by-products contain bacterial biomass and gases and they are ultimately excreted. Researches proved that inulin is appropriate for diabetic use higher doses of inulin benefit them [19]. This is because inulin secretion and glucagon secretion do not affect on inulin intake.

\section{Bifidogenic or prebiotic effect}

The growth and metabolic activity of certain bacteria in the colon, especially bifidobacteria and lactobacilli, is stimulated by inulin, thus promoting their health and this is known as a bifidogenic or prebiotic effect. Absorption resistance in the upper GIT and fermentation resistance in the intestine by microflora is must be graded as prebiotic. Probiotics are living micro-organisms that have beneficial effects on the host by restoring the gut's microbial composition [20]. Prebiotics, however, are indigestible elements of food that have beneficial effects by increasing the activity and development of one or more colonic bacteria. Food supplementation with Inulin can be allowed to increase bifidobacteria count and thus boost the microbiota of the gut. As a result of higher microbial activity, inulin absorption in various products strengthened their firmness. The researchers concluded that, when carbohydrates which are derived from tubers, sored in cool temperatures for 2 to 4 mo, displayed the effective in vitro prebiotic activity [21], much higher than commercial inulin. The capacity to survive into the distal area of the colon is another desired trait for prebiotics. This is where 
most chronic diseases, including colon cancer and ulcerative colitis, originate.

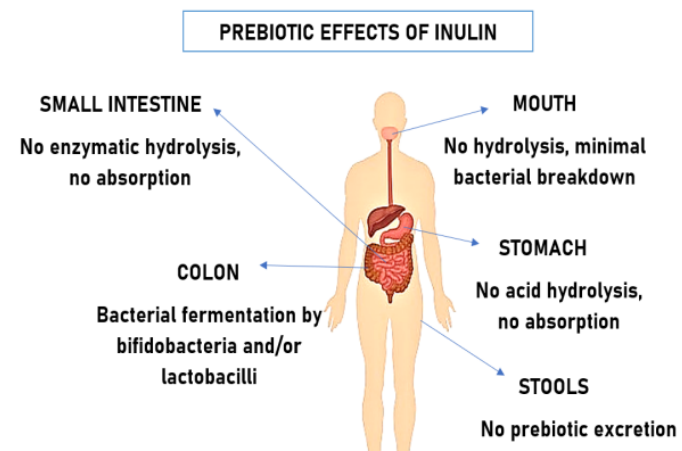

Fig. 5: Prebiotic effects of inulin [21]

\section{As a dietary fibre}

Their acts as dietary fibres are another most significant feature of inulin. owing to its health-promoting qualities, exposure to dietary fiber-enriched diets has been increased. Inulin and oligofructose affect intestinal function by raising the frequency of stools, especially in the case of constipation [22]. The stool weight off inulin has been increased to $2 \mathrm{~g}$ for each gram of inulin. Additionally, they reportedly decrease serum triglycerides and blood cholesterol levels in hypercholesterolemic patients. Besides, the inulin intake made serum triglycerides and blood cholesterol levels to decrease in hypercholesterolemic patients. Fermentable by the microflora present in the large intestine without any changes in the small intestine is the fundamental characteristic of the dietary fibre.

\section{Antioxidant effect}

The risk of certain diseases may be reduced by a diet rich in antioxidants. Oxidation is a major biological mechanism that supplies the body's energy. Free radicals, when produced in high amounts, will cause severe damage to cells, tissues, and organs which leads to cancer, inflammation, and radiation damage. Antioxidants reduce the free radicals, thereby inhibiting the oxidation [23]. It is known that plant polysaccharides are rich in antioxidant activity and inulin is one among them. Based on the research conducted on the Caco-2 cell model, it is confirmed that intracellular antioxidant enzyme activities are enhanced by inulin [24]. The higher antioxidant activity is seen in the inulin having a lower degree of polymerization. In vivo studies on laying hens confirmed the antioxidant effect of inulin. The action of antioxidant enzymes increased with the increase in the concentration of the inulin. Inulin derivatives that are structurally modified showed improved activity than inulin [25]. The activity of antioxidants was associated with the rate and position of the amino groups.

\section{Minimize the chance of colon cancer and IBD}

Inulin has favorable properties to decrease the risk of many intestinal tract diseases, particularly IBD (irritable bowel diseases) and colon cancer. A commonly diagnosed tumor in the colon cells caused by mutations in colon cancer and is also known as rectal cancer or colorectal cancer. Inflammatory bowel disorder is a mixture of ulcerative colitis and Crohn's disease. Both of these are chronic GIT inflammatory diseases that affect up to 250 per 50,000 people in the world today.

Colon cancer is one of the fast-growing malignant tumors which is caused by human lifestyle changes such as dietary changes. Many scientists are still working on the production of safe, low-toxic agents for treating colon cancer from natural origin. There is growing evidence that plant polysaccharides can effectively reduce the risk of colon cancer. Aberrant crypt foci formation is a primary sign of colon cancer. Researches prove that high inulin intake can significantly inhibit the ACF formation thereby not occurring colon cancer [26]. Compared to oligofructose long-chain inulin can prevent the formation of ACF effectively. This is because of the requirement of more fermentation time for the long-chain inulin in the large intestine. Short-chain fatty acid development by fermentation in the intestine is another potential mechanism for inulin's anti-cancer impact [27].

The prevalence of IBD has risen dramatically over the past few decades. Recent research has shown that a combination of a variety of genetic, environmental, and immunological variables affects IBD. The combination of inulin and probiotic microorganisms has been observed to reduce colitis and enhance the development of intestinal bifidobacteria and lactobacilli. Besides, the combination of inulin and lactulose has been shown to minimize inflammation in colitis caused by dextran sodium sulfate (DSS). In a study, the use of a $15 \mathrm{~g}$ combination of oligofructose and inulin for $21 \mathrm{~d}$ reduced Crohn's disease and increased intestinal bifidobacteria development. The combination of inulin and oligofructose inhibits IBD by enhancing the gut mucosal barrier. Inulin and oligofructose both improve the development of short chain fatty acids and favor the production of Lactobacilli or Bifidobacteria.

\section{Effect of inulin on obesity}

Obesity is a general condition with an excess amount of fat in the body. It is a medical condition that raises the risk of other illnesses such as heart disease, diabetes, high blood pressure, and some cancers, as well as other health issues. It is found that by the 21st century, obesity is going to become the greatest killer that threatens human health and life satisfaction. Inulin is a dietary fiber that is not decomposed in the digestive tract, it is sometimes used as a replacement for sucrose which helps in weight loss. Many human trials and animal trials have been conducted to prove the potential of inulin on obesity [28]. A human study demonstrated that intake of inulin in combination with green tea, which is high in catechins, is helpful for bodyweight maintenance and it also lowers blood pressure to avoid more cardiovascular diseases. The inulin-enriched diet substantially increased the feeling of fullness and decreased the intake of energy [29]. In a study conducted by Anastasovska et al., found that the function of hypothalamic neurons can be significantly altered by inulin. It is understood that inulin's mechanism for minimizing the obesity risk may be linked to improvements in the development of intestinal flora and fat oxidation promotion.

\section{Impact on diabetes}

Inulin is the most suitable food for diabetic patients. Continuous inulin intake for a couple of weeks will substantially lower HbA1c levels, an important component for blood sugar measurement [30]. The circulating anorexia intestinal hormone can be increased by inulin, which is a piece of key evidence for inulin to reduce blood sugar. Studies on inulin showed the hypoglycemic effect of inulin in animal models [31]. Inulin when given with reishi mushroom also known as Ganoderma lucidum, a plant polysaccharide, improves the metabolism of lipids as well as glucose which is a crucial thing in diabetes. L. plantarum in combination with inulin have been identified to minimize hyperglycemia and hyperlipidemia, also in lowering oxidative stress and to raise insulin as well as leptin levels in the T2DM rat's hypothalamus.

\section{Impact on constipation and stool frequency}

Constipation is surely the most common gastrointestinal issue among older people. Inulin and oligofructose increase the frequency and consistency of stools. Consuming every gram of inulin increase the weight of wet fecal up to 1-2g [32]. In participants with reduced initial stool frequency, the enhancement is greater. Inulin, a soluble fiber that cannot be digested by human enzymes and has an effect on the efficacy of the stomach, thereby reducing the intestine $\mathrm{pH}$, relieve constipation, and increases stool rate. Taking inulin-rich foods and high-water intake will relieve the constipation problem. Taking 25-35 g of inulin a day has been shown to alleviate constipation [33]. 


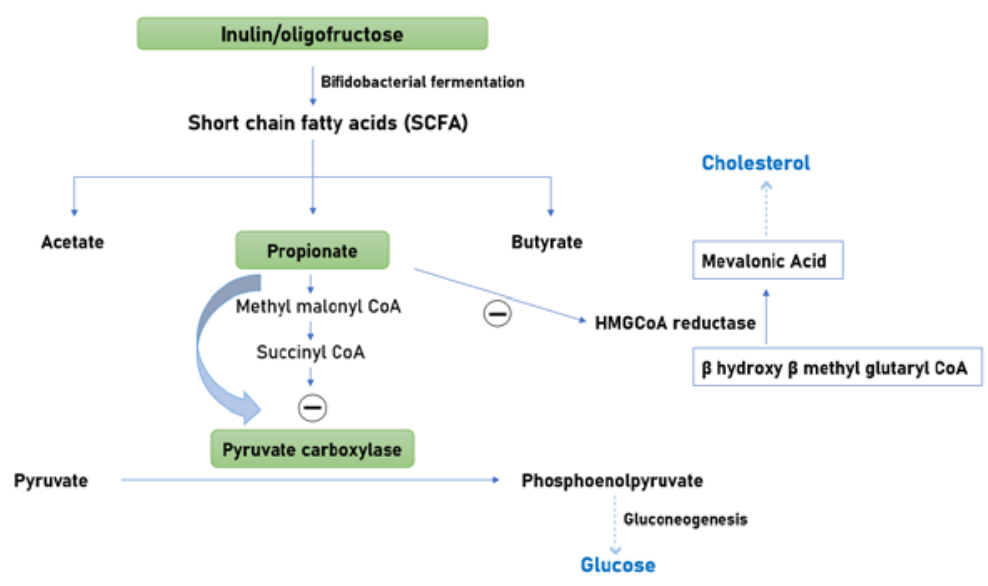

Fig. 6: Inhibition of pyruvate carboxylase by inulin [30]

\section{Impact on lipid metabolism}

Lipid metabolism, in short, is known as the degradation of lipids in cells. Inulin's nondigestible feature favours in reducing the risk of high concentrations of triacylglycerol. Inulin-derived oligofructose can significantly decrease triglycerides in both liver and serum, increase the ratios of lipoproteins, and also reduces cholesterol. The daily intake of 10 grams of inulin decreases the risk of atherosclerosis by lowering blood lipogenesis and triacylglycerol plasma concentrations. Various human studies show that inulin is more successful than oligofructose in reducing triglyceridemia. Several studies on rats, rodents proved the effect of inulin in reducing the production of genes that are responsible for the lipogenesis of an enzyme [34]. In addition, it can combat the accumulation of triacylglycerol in the liver and has beneficial effects on hepatic steatosis. The various mechanisms by which inulin influences the metabolism of lipids include: Elevated activity of the muscle LPL enzyme, increased production of satiogenic peptide and polyamine, increased ornithine decarboxylase activity, Increased Bifidobacteria population, and short-chain fatty acid (SCFA) production, etc. SCFAs are used as an energy source and appear to promote the health of the intestinal mucosa [35].

\section{Immune system simulation}

The immune system is very essential for the body to conduct immune responses and many functions, consisting of immune organs (IO), immune cells (IC), and immune molecules (IM). The immune system has the role of recognizing and excluding antigenic foreign bodies as the most significant protection system in the human body is to defend against pathogens. Different complex disorders [36]. Such as allergic diseases, AIDS, rheumatoid arthritis, etc., will occur until the immune system is weakened. In order to keep atmospheric equilibrium and physiological harmony, the immune mechanism coordinates with other body systems. The immune system in the human body is probably highly complex. The complexity of the immune system helps it to respond to foreign substances and prevents pathogenic species from intrusion, thereby defend the body by detecting and acting on antigens likely harmful substances. Immune-modulating effect of inulin on gut-associated lymphoid tissue (GALT) was investigated as early as 2002 and indicated that the probable mechanism would include shifts in intestinal flora induced by inulin's fermentation [37]. Several animal studies have shown that $\mathrm{T}$ cell functions, Natural killer cells, and phagocytic activities are indirectly activated by inulin and oligofructose by altering the intensity of lactic acid bacteria in the GIT, which not only protects mice from infections, as well as from tumours [38]. Besides, combined administration of galactooligosaccharides and inulin can enhance immune responses and improve the role of intestinal shielding in neonatal and postweaning mice. Given in combination, inulin, and oligo-fructose in mice showed the rate of protection against vaccination increased from $74 \%$ to $40 \%$. The rate of growth and function of immune-related genes were significantly improved in diets enriched with inulin. A diet enriched with inulin and oligofructose is given for three to four months before immunization against the influenza virus and observed that the NK cell function was increased by the dietary supplements [39]. In addition, fewer illnesses were found to be encountered by volunteers who followed the dietary supplement for a year.

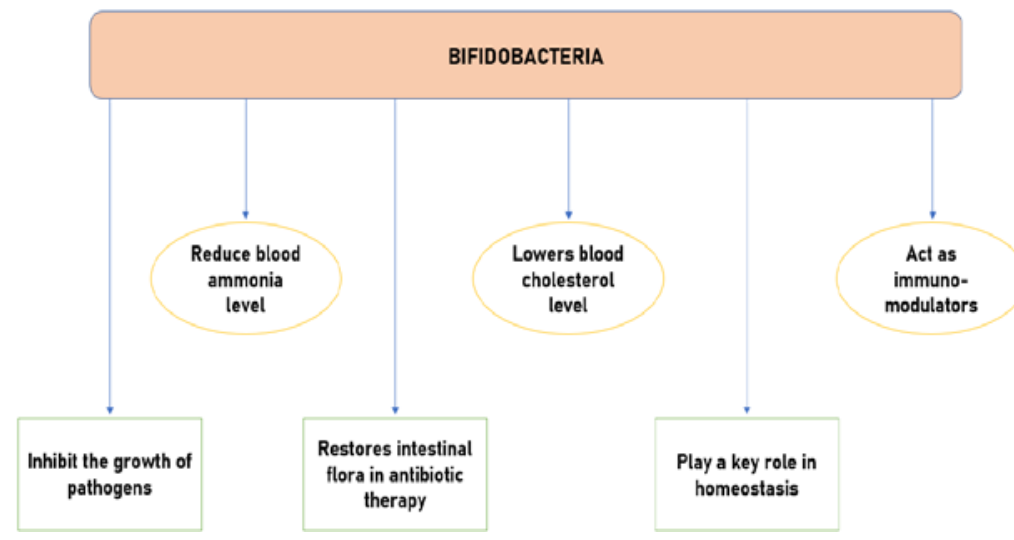

Fig. 7: Health promoting properties of bifidobacterial [30]

\section{Improve mineral absorption}

Minerals help your body grow, improve, and stay healthy, just like vitamins. From constructing powerful bones to transmitting nerve impulses, the body uses minerals to perform several different functions. To make hormones or maintain a regular heartbeat, some minerals are also used. Mineral absorption happens mostly in the proximal portion of the small intestine. The recommended minimum 
dietary intake of magnesium ranges from $340-420 \mathrm{mg} /$ day for males and from $2700-320 \mathrm{mg} /$ day for females. It accounts for $750-800 \mathrm{mg}$ per day in males in the case of calcium, while 800-1000 mg per day in females. Several research studies have reported that inulin can improve the absorption of minerals, particularly minerals like calcium and magnesium[40]. The reduction of intestinal $\mathrm{pH}$ may be one way, as colonic inulin fermentation produces SCFA or organic acids resulting in lower $\mathrm{pH}$ of the large intestine. During fermentation by intestinal flora, inulin releases SCFA, leading to decreased luminal $\mathrm{pH}$ resulting in higher calcium concentration and accelerating passive calcium diffusion. The fermented inulin product can also promote colonic mucosal growth and thus increase the area of intestinal absorption. Animal research studies have shown that inulin fructans have significantly improved mineral absorption, mainly calcium and magnesium [41]. In humans, fructan-type inulin has very little effect on mineral absorption in the small intestine.

\section{Intestinal acceptability}

The intestinal suitability of nondigestible constituents has been demonstrated in two ways [42]. One is the osmotic effects of the indigestible components result in increased water content in the colon. Higher osmotic pressure is caused by small chemicals, and excess water flows through the colon. For this cause, sorbitol and lactulose have a much greater purgative effect, unlike inulin. the other way to demonstrate intestinal acceptability is there are some effects, mainly gas output, caused by fermented goods. Components that require more fermentation time are better compared to components that ferment quickly. This is why inulin is easy to survive. Flatulence is a documented and frequently assumed dietary fiber intake after effects. Inulin showed strong tolerance without any after-effects in a study of giving $10 \mathrm{~g}$ of it per day [43].

\section{Role of Inulin in the pharmaceutical industry}

\section{For targeted drug delivery}

\section{For Colon-targeting}

It is difficult to treat colon diseases since oral administered medications are absorbed at either the level of the stomach and small intestine and do not enter the colon [44]; however, intravenously administered drugs are withdrawn from the body before reaching the colon. For colon disease treatment, the development of colon-targeted therapeutic agents is therefore essential. Inulin is specifically hydrolyzed as a natural polysaccharide by inulinase produced by bifidobacteria and not hydrolyzed by the enzymes present in GIT. Inulin and its derivatives are classified as biodegradable colon-targeted delivery vehicles based on the above information. Different inulin and dextran hydrogels were designed to act as possible carriers for drug delivery into the colon. Since inulin is not consumed by the stomach or SI, and as inulin is destroyed by colonic bacteria, drugs encapsulated in vesicles coated with inulin may be directly released into the colon. Thus, improvements in the treatment of colonic diseases include the use of inulin-coated vesicles. The drug delivery mechanism unique to the colon should be capable of protecting the drug en route to the colon [45]. The reason that inulin in the intestinal tract is not digested or consumed has made this polymer an attractive vehicle for the delivery of colonic drugs. Several types of drug deliveries, such as tablets, microspheres, microparticles, nanoparticles, micelles, nano micelles, hydrogels, have been designed and shown promising results with inulin and its derivatives. Some of the dosage form/drug deliveries for colon targeting with the aid of inulin are shown in table 1.

\section{To optimize drug activity for tumor-targeting}

Due to its excellent biocompatibility, biodegradability, and flexibility for simple chemical design, inulin has been broadly used as a linear organic polysaccharide in the preparation of tumor-targeting drug delivery systems. Doxorubicin (DOX) and Paclitaxel (PTX) were found more effective up to 100-200 times when they are loaded in nano-micelles of lauryl carbamate derivatives of the inulin compared to those drugs insoluble form. In further studies, epirubicin in free form exhibit less antitumor activity compared to inulin-ibuprofen polymer-based nanoparticles loaded with epirubicin [46]. The nanoparticles also exhibited lower toxicity. The numerous effective dosage formulations designed to treat tumors with the aid of inulin are shown in table 1.

Table 1: Applications of inulin in drug delivery

\begin{tabular}{llll}
\hline & & Colon targeting & \\
\hline Inulin or inulin derivatives & Model drug & Dosage forms & References \\
\hline Inulin & Infliximab & Tablet & {$[47]$} \\
Cinnamoylated inulin & Methotrexate & Microspheres & {$[44]$} \\
Inulin acetate & Indomethacin & Microparticles & {$[48]$} \\
Amphiphilic inulin & Ornidazole & Nanoparticles & {$[49]$} \\
Methacrylated inulin & Prednisolone & Gels & {$[50]$} \\
Methacrylated inulin & Bovine serum & Hydrogels & {$[51]$} \\
Lipoic acid esterified inulin & Tanshinone IIA & Micelles & {$[51]$} \\
PEGylated squalene-grafted-inulin & Doxorubicin & Superparamagnetic iron oxide nano-particle (SPIONs) & {$[52]$} \\
Invite-Bio & -- & Long circulated nano micelles & {$[53]$} \\
Tumor targeting & & & \\
Inulin-Ibuprofen polymer & Epirubicin & Nanoparticles (Neuroblastoma) & {$[12]$} \\
Inulin-2-aminoethyl carbamate & Doxorubicin & Gold nanoparticles (Cervical and lung cancer) & \\
Carboxymethyl inulin & 5-Fluoro uracil & Silver nanoparticles (Pancreatic cancer) & {$[54]$} \\
Inutec® SP1 & Paclitaxel & Micelles (Skin cancer) & {$[55]$} \\
Inutec® SP1 & Doxorubicin and Paclitaxel & Nano micelles (Breast cancer) & {$[56]$} \\
Inulin-cysteamine- & siRNA & Superparamagnetic iron oxide nano-particle (SPIONs) (Breast & {$[57]$} \\
Diethylenetriamine copolymer & & cancer) & \\
\end{tabular}

\section{As a stabilizer for different drug deliveries}

\section{Protein-based drugs}

The discovery of a variety of protein-based therapeutic agents has resulted in rapid developments in microbiology and biopharmaceutical technologies. In aqueous solutions, as these are unstable, so they are available as powders that have been dried through freeze-drying or spray drying, or evaporative drying technique. This may significantly affect either the action or efficacy [59]. Therefore, in these drying processes, a protective agent needs to be applied to prevent the harmful effects. During the drying and subsequent storage period, it is known that sugars can protect proteins. The detailed mechanisms are, however, not well understood. A study on the enzyme stabilizing effects was conducted as early as 1992, revealing that inulin does not have excellent stabilizing effects compared to sugar, sorbitol, and dextran [60]. The poor stabilizing result may be due to the degree of polymerization and less group content.

A significant count of recent studies shown that inulin has a strong stabilizing effect, especially on protein-based drugs. As a model 
protein, alkaline phosphatase was used to research whether inulin is a competent protein stabilizer. The results showed that the behavior of alkaline phosphatase in drying and storage for $4 \mathrm{w}$ at $20^{\circ} \mathrm{C}$ with relative humidity at 0,45 , or 60 can be perfectly maintained by inulin as a stabilizer. Inulin has been reported to be a competent stabilizer of haemagglutinin, an essential component of the influenza vaccine. The strength of haemagglutinin and the immunogenicity of virosomes are preserved by inulin during lyophilization and long- term storage. Inulin demonstrated outstanding protein safety, and it also demonstrated its superiority over trehalose in preserving stability after the tableting process. In a study conducted by Tonniset al. Inulin with $4 \mathrm{kDa}$ showed the best protective effect compared to dextran with $6 \mathrm{kDa}$, this indicates that the size and molecular flexibility of sugars influence their protein stabilization ability [61]. Therefore, inulin is an effective stabilizer for proteinbased drugs.

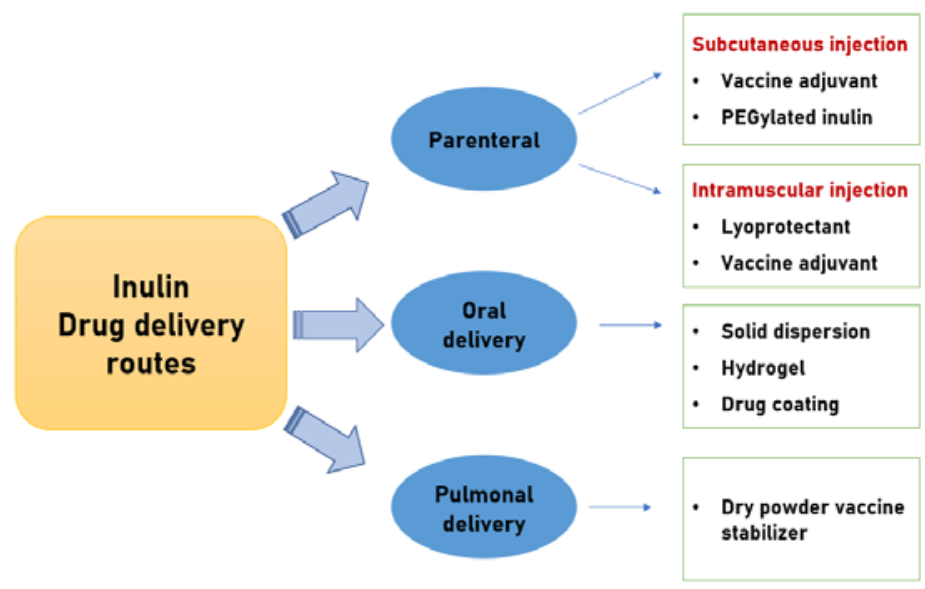

Fig. 8: Various routes of Inulin delivery [17]

\section{Other systems of drug delivery}

Inulin can also be a product stabilizer in other dosage types such as Solid dispersions, PEGylated nanoparticles, solid lipid nanoparticles, etc, in parallel to its function as a protein-based drug stabilizer. Solid dispersions obtained by suspending inulin and tetrahydrocannabinol in THC (tertiary butyl alcohol) and water demonstrated high stability over uncoated THC during storage at $20-25^{\circ} \mathrm{C}$ and $45 \%$ RH for $250 \mathrm{~d}$. In addition, studies have reported that solid dispersions prepared by spray drying containing THC and IN showed better stability than prepared by freeze-drying. Inulin with 1.5 and $4 \mathrm{kDa}$ is proved to be an effective protectant for PEG-modified liposomes. Due to its excellent compatibility with PEG, inulin is a superior stabilizer over dextran for various forms of PEGylated nanoparticles. A variety of structurally modified inulin is often used commonly as stabilizers at present. In the solid lipid nanoparticles, dodecyl inulin and octadecyl inulin were used as a surfactant. These prevented the transfer of lipid crystals and shielded particles from aggregation throughout storage and were superior to the traditional surfactants such as Tweens and Poloxamer.

\section{Enhancement in the action of the drug}

\section{Enhancement of drug release}

For solid oral formulations, before it can be absorbed, the medication must be dissolved. Thus, the drugs having poor dissolution as well as poor release profiles exhibit poor bioavailability [62]. The dissolution and release profile of these drugs can be enhanced by techniques such as solid dispersion. The use of hydrophilic carriers in the solid dispersion makes it dissolve at a faster rate, thereby increasing bioavailability.

And as inulin exhibits hydrophilic properties, it is used as carriers in the dosage forms which gradually increases the drug dissolution, release, and bioavailability. In a study conducted on the dissolution rate of diazepam, inulin significantly improved the dissolution profiles compared with sucrose or other sugars. Further, a study showed the excellent improvement in the dissolution of diazepam by using solid dispersion tablets containing inulin DP23. Only 10-15 percent of the drug is released from physical mixtures, whereas $80-85$ percent of the release was seen from solid dispersion containing inulin. In a comparative study of fenofibrate's dissolution and stability profile using different carriers like PEG 20k, HP- $\beta$-CD, PVP K30, mannitol, and inulin $4 \mathrm{kDa}$, the inulin carrier exhibited excellent dissolution and better stability. Inulin derivatives exhibited controlled release properties in the studies conducted on Irbesartan. Within $60 \mathrm{~min}$, the dissolution rate of Irbesartan reached 90\%, while poly (acrylic acid) grafted inulin can achieve approximately $33 \%$ controlled-release of Irbesartan.

Moreover, multicomponent solid dispersions prepared by excipients such as mannitol, inulin, and cellulose acetate phthalate not only boost irinotecan (IRN) dissolution and also increase the permeability through the intestine. The various effective dosage forms designed for improved solubility and stability with the aid of inulin are shown in table 2.

Table 2: Various applications of inulin

\begin{tabular}{llll}
\hline Inulin or its derivatives & Model drug & Dosage form & Applications \\
\hline Inulin & Fiseten & Inulin nano-particles & Improves solubility and stability \\
Inulin-D- $\alpha$-tocopherol succinate & Curcumin & Self assembling micelles & Improves solubility \\
\cline { 2 - 3 } Inulin & Anthocyanins & Microcapsules & Improves stability \\
Hydrophobically Modified inulin & Cinnamaldehyde & Nanoemulsions & Improves solubility of nanoparticles \\
Aminated inulin & Folic acid complex & Modified release & Controlled \\
\hline
\end{tabular}

\section{As a diagnostic tool}

In measuring GFR

Inulin can only be cleared by the kidneys in the human body. It is understood that inulin will be filtered from the glomerulus without being reabsorbed by renal tubules. Inulin is thus defined as the most precise substance for clinically measuring glomerular filtration rate (GFR) to evaluate renal function. In this application, inulin is injected intravenously [67]. Although inulin is commonly accepted as the benchmark for GFR clinical determination, this technique has 
complex procedures and is not sufficient for routine clinical measurement. Researchers have recently upgraded the technique and developed near-IR-dye-labeled inulin as an optical probe to test GFR by transcutaneous pulse dye densitometer, accurately [68]

\section{Vaccine adjuvants}

Vaccine adjuvants are the agents that increase certain immune responses for a particular antigen. Aluminum salts are the only vaccine adjuvants currently being approved by the FDA. Others all are considered experimental and need to be subjected to special preclinical examinations [69]. They can be paired with unique vaccines that can offer many benefits, including minimizing the use of antigens, easily stimulating the immune system, strengthening the immune response, and extending the continuous release of antigens. In the early 1980's itself, there were studies that exhibited $\gamma$-Inulin's adjuvant activity. Researchers have recently verified that $\delta$-inulin, which has comparable solubility to $\gamma$-inulin, has excellent adjuvant activity as well [70]. Advax, a microparticle derived from $\delta$-IN, has been developed as an adjuvant for many vaccines, such as influenza, hepatitis B, SARS, anthrax, HIV, etc.

\section{CONCLUSION}

Consumers are more mindful of their diet in modern times and demand a low caloric diet, thus improving well-being. Inulin is one such dietary food in the modern era that has garnered attention. This review thoroughly described the biological processes of inulin, including the effect of prebiotics, antioxidants, controlling the metabolism of sugars or lipids, Enhancing the mineral absorption, reducing the risk of IBD, colon cancer, and obesity as a stabilizer for drugs, etc. Inulin has been recognized as having tremendous effects on human growth and state of health. Besides, it can be a good carbohydrate and fat substitute and a strong source of fibre to enrich various foods for product growth. A significant volume of literature indicates that in pharmaceuticals, inulin has great promising applications. For some drugs, especially the proteinbased, it can be used as a stabilizer, can greatly enhance dissolution rate and drug release, it can also be used for controlled-release and targeted preparationsFurthermore, studies on chemically modified inulin are increasingly becoming an active field of study. Chemically modified inulin showed great characteristics that made up for the inadequacy of inulin, which further broadened inulin applications. Though the processing technology of inulin is very advanced and can achieve high volume, there are still some challenges for separating and purifying inulin with a single degree of polymerization. Complex systems, weak operability, and low yield are among these obstacles. Research on the separation and purification of inulin is therefore still a field that requires further focus.

\section{FUNDING}

Nil

\section{AUTHORS CONTRIBUTIONS}

All authors have contributed equally.

\section{CONFLICT OF INTERESTS}

The author has no conflict of interest to declare.

\section{REFERENCES}

1. Niness KR. Inulin and oligofructose: what are they? J Nutr 1999;129:1402-6.

2. Clark DE. Rapid calculation of polar molecular surface area and its application to the prediction of transport phenomena. Prediction of intestinal absorption. J Pharm Sci 1999;88:807-14.

3. Franck A. Technological functionality of inulin and oligofructose. Br J Nutr 2002;87:S287-91.

4. Watzl B, Girrbach S, Roller M. Inulin, oligofructose and immunomodulation. Br J Nutr 2005;93:S49-55.

5. Roberfroid MB. Introducing inulin-type fructans. Br J Nutr 2005;93:S13-25.

6. Shoaib M, Shehzad A, Omar M, Rakha A, Raza H, Sharif HR, et al. Inulin: properties, health benefits and food applications. Carbohydr Polym 2016;147:444-54.
7. Jimenez Sanchez M, Perez Morales R, Goycoolea FM, Mueller M, Praznik W, Loeppert R, et al. Self-assembled high molecular weight inulin nanoparticles: Enzymatic synthesis, physicochemical and biological properties. Carbohydr Polym 2019;215:160-9.

8. Roberfroid MB, Van Loo JAE, Gibson GR. The bifidogenic nature of chicory inulin and its hydrolysis products. J Nutr 1998;128:11-9.

9. Roberfroid MB. Functional foods: concepts and application to inulin and oligofructose. Br J Nutr 2002;87:S139-43.

10. Flamm G, Glinsmann W, Kritchevsky D, Prosky L, Roberfroid M. Inulin and oligofructose as dietary fiber: a review of the evidence. Crit Rev Food Sci Nutr 2001;41:353-62.

11. Ni D, Xu W, Zhu Y, Zhang W, Zhang T, Guang C, et al. Inulin and its enzymatic production by inulosucrase: Characteristics, structural features, molecular modifications and applications. Biotechnol Adv 2019;37:306-18.

12. Wan X, Guo H, Liang Y, Zhou C, Liu Z, Li K, et al. The physiological functions and pharmaceutical applications of inulin: a review. Carbohydr Polym 2020;246:116589.

13. Roberfroid M. Dietary fiber, inulin, and oligofructose: a review comparing their physiological effects. Crit Rev Food Sci Nutr 1993;33:103-48.

14. Devine DA, Marsh PD. Prospects for the development of probiotics and prebiotics for oral applications. J Oral Microbiol 2009;1:1-12.

15. Gupta N, Jangid AK, Pooja D, Kulhari H. Inulin: a novel and stretchy polysaccharide tool for biomedical and nutritional applications. Int J Biol Macromol 2019;132:852-63.

16. Kim Y, Faqih MN, Wang SS. Factors affecting gel formation of inulin. Carbohydr Polym 2001;46:135-45.

17. Imran S, Gillis RB, Kok MS, Harding SE, Adams GG. Application and use of inulin as a tool for therapeutic drug delivery. Biotechnol Genet Eng Rev 2012;28:33-46.

18. Slavin J. Fiber and prebiotics: mechanisms and health benefits. Nutrients 2013;5:1417-35.

19. Carabin IG, Gary Flamm W. Evaluation of safety of inulin and oligofructose as dietary fiber. Regul Toxicol Pharmacol 1999;30:268-82.

20. Gibson GR. Dietary modulation of the human gut microflora using the prebiotics oligofructose and inulin. J Nutr 1999;129:1438-41.

21. Kolida S, Tuohy K, Gibson GR. Prebiotic effects of inulin and oligofructose. Br J Nutr 2002;87:193-7.

22. Karimi R, Azizi MH, Ghasemlou M, Vaziri M. Application of inulin in cheese as prebiotic, fat replacer and texturizer: a review. Carbohydr Polym 2015;119:85-100.

23. Liu J, Lu J Feng, Wen X Yuan, Kan J, Jin C Hai. Antioxidant and protective effect of inulin and catechin grafted inulin against CCl4induced liver injury. Int J Biol Macromol 2015;72:1479-84.

24. Xiong $\mathrm{X}$, Huang G, Huang $\mathrm{H}$. The antioxidant activities of phosphorylated polysaccharide from native ginseng. Int J Biol Macromol 2019;126:842-5.

25. Pasqualetti V, Altomare A, Guarino MPL, Locato V, Cocca S, Cimini S, et al. Antioxidant activity of inulin and its role in the prevention of human colonic muscle cell impairment induced by lipopolysaccharide mucosal exposure. PLoS One 2014;9:1522.

26. Ingredients F, Roberfroid MB. Inulin-type fructans. Functional 2018;4:2493-502.

27. Pool Zobel BL. Inulin-type fructans and reduction in colon cancer risk: review of experimental and human data. Br J Nutr 2005;93:73-90.

28. Man S, Liu T, Yao Y, Lu Y, Ma L, Lu F. Friend or foe? The roles of inulin-type fructans. Carbohydr Polym 2021;252:117-55.

29. Yang HY, Yang SC, Chao JCJ, Chen JR. Beneficial effects of catechin-rich green tea and inulin on the body composition of overweight adults. Br J Nutr 2012;107:749-54.

30. Kaur N, Gupta AK. Applications of inulin and oligofructose in health and nutrition. J Biosci 2002;27:703-14.

31. Liu J, Lu J Feng, Kan J, Wen X Yuan, Jin C Hai. Synthesis, characterization and in vitro anti-diabetic activity of catechin grafted inulin. Int J Biol Macromol 2014;64:76-83.

32. Gibson GR, Beatty ER, Wang XIN, Cummings JH. Oligofructose and inulin. Gastroenterology 1995;1:975-82. 
33. Fernandez Banares F. Nutritional care of the patient with constipation. Best Pract Res Clin Gastroenterol 2006;20:575-87.

34. Alles MS, De Roos NM, Bakx JC, Van De Lisdonk E, Zock PL, Hautvast JGAJ. Consumption of fructooligosaccharides does not favorably affect blood glucose and serum lipid concentrations in patients with type 2 diabetes. Am J Clin Nutr 1999;69:64-9.

35. Roberfroid MB. Nutritional and health benefits of inulin and oligofructose caloric value of inulin and oligofructose. J Nutr 1999;129:1436-7.

36. Al-Taani B, Khanfar MAI, Alsuod Oabu. Enhancement of the release of curcumin by the freeze-drying technique using inulin and neusilin as carriers. 2018;10:3-9.

37. Banerjee D, Chowdhury R, Bhattacharya P. The prebiotic influence of inulin on growth rate and antibiotic sensitivity. 2016;8:181-4.

38. Bunout D, Barrera G, Hirsch S, Gattas V, De La Maza MP, Haschke $\mathrm{F}$, et al. Effects of a nutritional supplement on the immune response and cytokine production in free-living Chilean elderly. J Parenter Enter Nutr 2004;28:348-54.

39. Kelly Quagliana KA, Nelson PD, Buddington RK. Dietary oligofructose and inulin modulate immune functions in mice. Nutr Res 2003;23:257-67.

40. Scholz Ahrens KE, Schrezenmeir J. Inulin, oligofructose and mineral metabolism - experimental data and mechanism. Br J Nutr 2002;87:S179-86.

41. Coudray C, Bellanger J, Castiglia Delavaud C, Remesy C, Vermorel M, Rayssignuier Y. Effect of soluble or partly soluble dietary fibres supplementation on absorption and balance of calcium, magnesium, iron and zinc in healthy young men. Eur J Clin Nutr 1997;51:375-80.

42. Coussement PAA. Nutritional and health benefits of inulin and oligofructose inulin and oligofructose: safe intakes and legal status. J Nutr 1999;129:1412-7.

43. Ripoll C, Flourie B, Megnien S, Hermand O, Janssens M. Gastrointestinal tolerance to an inulin-rich soluble roasted chicory extract after consumption in healthy subjects. Nutrition 2010;26:799-803.

44. Lopez Molina D, Chazarra S, How CW, Pruidze N, Navarro Peran E, Garcia Canovas F, et al. Cinnamate of inulin as a vehicle for delivery of colonic drugs. Int J Pharm 2015;479:96-102.

45. Koruri SS, Banerjee D, Chowdhury R. Studies on prebiotic food additive (inulin) in indian dietary fibre sources-garlic (allium sativum), wheat (triticum spp.), oat (avena sativa) and dalia (bulgur). Int J Pharm Pharm Sci 2014;6:278-82.

46. Varan G, Varan C, Bilensoy E. Plant based natural polymeric nanoparticles as promising carriers for anticancer therapeutics. Polymeric nanoparticles as a promising tool for anti-cancer therapeutics. Elsevier Inc.; 2019. p. 293-318.

47. Maurer JM, Hofman S, Schellekens RCA, Tonnis WF, Dubois AOT, Woerdenbag HJ, et al. Development and potential application of an oral ColoPulse infliximab tablet with colon specific release: a feasibility study. Int J Pharm 2016;505:175-86.

48. Shivhare K, Garg C, Priyam A, Gupta A, Sharma AK, Kumar P. Enzyme sensitive smart inulin-dehydropeptide conjugate selfassembles into nanostructures useful for targeted delivery of ornidazole. Int J Biol Macromol 2018;106:775-83.

49. Maris B, Verheyden L, Van Reeth K, Samyn C, Augustijns P, Kinget $\mathrm{R}$, et al. Synthesis and characterisation of inulin-azo hydrogels designed for colon targeting. Int J Pharm 2001;213:143-52.

50. Van Den Mooter G, Vervoort L, Kinget R. Characterization of methacrylated inulin hydrogels designed for colon targeting: in vitro release of BSA. Pharm Res 2003;20:303-7.

51. Wang D, Sun F, Lu C, Chen P, Wang Z, Qiu Y, et al. Inulin-based glutathione-responsive delivery system for colon cancer treatment. Int J Biol Macromol 2018;111:1264-72.

52. Maksimenko A, Dosio F, Mougin J, Ferrero A, Wack S, Reddy LH, et al. A unique squalenoylated and nonpegylateddoxorubicin nanomedicine with systemiclong-circulating properties and anticancer activity. Proc Natl Acad Sci USA 2014;111:217-26.
53. Mandracchia D, Rosato A, Trapani A, Chlapanidas T, Montagner IM, Perteghella S, et al. Design, synthesis and evaluation of biotin decorated inulin-based polymeric micelles as longcirculating nanocarriers for targeted drug delivery. Nanomed Nanotechnol Biol Med 2017;13:1245-54.

54. Mauro N, Campora S, Scialabba C, Adamo G, Licciardi M, Ghersi $\mathrm{G}$, et al. Self-organized environment-sensitive inulindoxorubicin conjugate with a selective cytotoxic effect towards cancer cells. RSC Adv 2015;5:32421-30.

55. Nigam Joshi P, Agawane S, Athalye MC, Jadhav V, Sarkar D, Prakash R. Multifunctional inulin tethered silver-graphene quantum dots nanotheranostic module for pancreatic cancer therapy. Mater Sci Eng C 2017;78:1203-11.

56. Muley P, Kumar S, El Kourati F, Kesharwani SS, Tummala H. Hydrophobically modified inulin as an amphiphilic carbohydrate polymer for micellar delivery of paclitaxel for intravenous route. Int J Pharm 2016;500:32-41.

57. Kesharwani SS, Dachineni R, Bhat GJ, Tummala H. Hydrophobically modified inulin-based micelles: transport mechanisms and drug delivery applications for breast cancer. J Drug Delivery Sci Technol 2019;54:101-254.

58. Sardo C, Craparo EF, Porsio B, Giammona G, Cavallaro G. Improvements in rational design strategies of inulin derivative polycation for siRNA delivery. Biomacromolecules 2016;17:2352-66.

59. Mensink MA, Frijlink HW, Van Der Voort Maarschalk K, Hinrichs WLJ. Inulin, a flexible oligosaccharide. II: a review of its pharmaceutical applications. Carbohydr Polym 2015;134:418-28.

60. Paolini G, Shapland R, Van Hoorn W. Global mapping of pharmacological space. Nat Biotechnol 2006;24:805-15.

61. Tonnis WF, Mensink MA, De Jager A, Van Der Voort Maarschalk K, Frijlink HW, Hinrichs WLJ. Size and molecular flexibility of sugars determine the storage stability of freeze-dried proteins. Mol Pharm 2015;12:684-94.

62. Leyva Porras C, Lopez Pablos AL, Alvarez Salas C, Perez Urizar J. Polysaccharides. Polysaccharides 2021;11:1-22.

63. Charoenwongpaiboon T, Wangpaiboon K, Panpetch P, Field RA, Barclay JE, Pichyangkura $\mathrm{R}$, et al. Temperature-dependent inulin nanoparticles synthesized by lactobacillus reuteri 121 inulosucrase and complex formation with flavonoids. Carbohydr Polym 2019;223:115-44.

64. Tripodo G, Pasut G, Trapani A, Mero A, Lasorsa FM, Chlapanidas $\mathrm{T}$, et al. Inulin- $\mathrm{d}-\alpha$-tocopherol succinate nanomicelles as a platform for effective intravenous administration of curcumin. Biomacromolecules 2015;16:550-7.

65. Hester SN, Mastaloudis A, Gray R, Antony JM, Evans M, Wood SM. Efficacy of anthocyanin and prebiotic blend on the intestinal environment in obese male and female subjects. J Nutr Metab 2018. https://doi.org/10.1155/2018/7497260

66. Cui H, Liu S, Lv Y, Wu S, Wang L, Hao F, et al. Transfer hydrogenation of cinnamaldehyde to cinnamyl alcohol in hydrophobically modified core-shell MOFs nanoreactor: identification of the formed metal- $\mathrm{N}$ as the structure of an active site. J Catal 2020;381:468-81.

67. Epstein JH. A photometric method for the determination in plasma and urine of It is generally accepted that, of the substances used to test glomerular filtration, inulin is the most satisfactory. Determination Inulin Clearance Inulin Injected Int 2015;13:839-46.

68. Hauser Kawaguchi A, Milne M, Li F, Lee TY, Luyt LG. The development of a near-infrared inulin optical probe for measuring glomerular filtration rate. Int J Biol Macromol 2019;123:255-60.

69. Skwarczynski M. Inulin: a new adjuvant with unknown mode of action. EBioMedicine 2017;15:8-9.

70. Cooper PD, Steele EJ. Algammulin, a new vaccine adjuvant comprising gamma inulin particles containing alum: preparation and in vitro properties. Vaccine 1991;9:351-7. 